PROCEEDINGS OF THE

AMERICAN MATHEMATICAL SOCIETY

Volume 139, Number 7, July 2011, Pages 2327-2336

S 0002-9939(2010)10675-5

Article electronically published on December 2, 2010

\title{
NEW CRITERIONS OF EXISTENCE AND CONJUGACY OF HALL SUBGROUPS OF FINITE GROUPS
}

\author{
WENBIN GUO AND ALEXANDER N. SKIBA
}

(Communicated by Jonathan I. Hall)

\begin{abstract}
In the paper, new criterions for existence and conjugacy of Hall subgroups of finite groups are given. In particular, the Schur-Zassenhaus theorem, Hall theorem and Čunihin theorem are generalized.
\end{abstract}

\section{INTRODUCTION}

Throughout this paper, all groups are finite, $G$ denotes a finite group and $\pi$ denotes a non-empty subset of the set of all primes. A subgroup $H$ is said to be permutable with a subgroup $B$ if $H B=B H$. The notation and terminology are standard, as in [10] and [3].

The famous Schur-Zassenhaus Theorem asserts that: If $G$ has a normal Hall $\pi$-subgroup $A$, then $G$ is an $E_{\pi^{\prime}}$-group (that is, $G$ has a Hall $\pi^{\prime}$-group). Moreover, if either $A$ or $G / A$ is soluble, then $A$ is a $C_{\pi^{\prime}}$-subgroup (that is, any two Hall $\pi^{\prime}$-subgroups of $G$ are conjugate).

In 1928, Hall [6] proved that: A finite soluble group has a Hall $\pi$-subgroup and any two Hall $\pi$-subgroups are conjugate in $G$.

In 1949, Cunihin developed further the Schur-Zassenhaus and Hall theorems and proved the following classical result.

Theorem (S. A. Cunihin [1]). If $G$ is $\pi$-separable, then $G$ is an $E_{\pi}$-group and an $E_{\pi^{\prime}}$-group. Moreover, if $G$ is $\pi$-soluble, then $G$ is a $C_{\pi}$-group and a $C_{\pi^{\prime}}$-group.

Note that a group $G$ is said to be $\pi$-separable if $G$ has a normal series

$$
1=G_{0} \leq G_{1} \leq \ldots \leq G_{t-1} \leq G_{t}=G,
$$

where each index $\left|G_{i}: G_{i-1}\right|$ is either a $\pi$-number or a $\pi^{\prime}$-number. A group $G$ is said to be $\pi$-soluble if each index $\left|G_{i}: G_{i-1}\right|$ of Series $(*)$ is either a $\pi$-prime power (that is, a power of some prime in $\pi$ ) or a $\pi^{\prime}$-number.

The example of the group $P S L(2,7)$ shows that the condition of normality for the members of Series $(*)$ could not be omitted.

Received by the editors February 23, 2010 and, in revised form, May 12, 2010 and June 19, 2010.

2010 Mathematics Subject Classification. Primary 20D10, 20D20.

Key words and phrases. Sylow subgroup, Hall subgroup, $\pi$-separable group, permutable subgroups, $C_{\pi}$-group.

The research of the first author was supported by an NNSF grant of China.

(C)2010 American Mathematical Society 
It is well known that the above Schur-Zassenhaus theorem, Hall theorem and Cunihin theorem are truly fundamental results of group theory. In connection with these important results, the following two problems have naturally arisen:

Problem I. Whether the conclusion of the Schur-Zassenhaus Theorem holds if the Hall subgroup $A$ of $G$ is not normal. In other words, can we weaken the condition of normality for the Hall subgroup $A$ of $G$ so that the conclusion of the SchurZassenhaus Theorem is still true?

Problem II. Whether we can replace the condition of normality for the members of Series $(*)$ by some weaker condition, for example, by permutability of the members of Series $(*)$ with some systems of subgroups of $G$.

Some results pertaining to Problem I have been obtained in [4, 5]. In Section 3 of this paper, we give the following further generalization of the Schur-Zassenhaus Theorem.

Theorem A. Let $A$ be a Hall $\pi$-subgroup of $G$. Let $G=A T$ for some subgroup $T$ of $G$, and let $q$ be a prime. If $A$ permutes with every Sylow p-subgroup of $T$, for all primes $p \neq q$, and either $A$ or $T$ is soluble, then $T$ contains a complement of $A$ in $G$ and any two complements of $A$ in $G$ are conjugate.

Notice that the well known Feit-Thompson theorem about solvability of groups of odd order is not used in the proof of Theorem A. By using the Feit-Thompson theorem, we obtain the following stronger version of Theorem A.

Theorem A*. Let $A$ be a Hall $\pi$-subgroup of $G$. Let $G=A T$ for some subgroup $T$ of $G$, and let $q$ be a prime. If $A$ permutes with every Sylow p-subgroup of $T$ for all primes $p \neq q$, then $T$ contains a complement of $A$ in $G$ and any two complements of $A$ in $G$ are conjugate.

Recall that a subgroup $H$ of $G$ is said to be a supplement of a subgroup $A$ in $G$ if $A H=G$. Let

$$
1=H_{0} \leq H_{1} \leq \ldots \leq H_{t-1} \leq H_{t}=G
$$

be some subgroup series of $G$. We say that a subgroup series

$$
1=T_{t} \leq T_{t-1} \leq \ldots \leq T_{1} \leq T_{0}=G
$$

is a supplement of Series (**) in $G$ if $T_{i}$ is a supplement of $H_{i}$ in $G$ for all $i=$ $0,1, \ldots, t$.

Another purpose of this paper is to give a positive answer to Problem II. We will prove the following results.

Theorem B. Suppose that $G$ has a subgroup series

$$
1=H_{0} \leq H_{1} \leq \ldots \leq H_{t-1} \leq H_{t}=G
$$

and a supplement

$$
1=T_{t} \leq T_{t-1} \leq \ldots \leq T_{1} \leq T_{0}=G
$$

of this series in $G$ such that $H_{i}$ permutes with every Sylow subgroup of $T_{i}$ for all $i=1,2, \ldots, t$. If each index $\left|H_{i+1}: H_{i}\right|$ is either a $\pi$-number or a $\pi^{\prime}$-number, then $G$ is an $E_{\pi}$-group and an $E_{\pi^{\prime}}$-group. Moreover, if each $\pi$-index $\left|H_{i+1}: H_{i}\right|$ is a prime power, then $G$ has a soluble Hall $\pi$-subgroup. 
Corollary 1.1. Suppose that $G$ has a subgroup series

$$
1=H_{0} \leq H_{1} \leq \ldots \leq H_{t-1} \leq H_{t}=G
$$

and a supplement

$$
1=T_{t} \leq T_{t-1} \leq \ldots \leq T_{1} \leq T_{0}=G
$$

of this series in $G$ such that $H_{i}$ permutes with all Sylow subgroups of $T_{i}$ for all $i=1,2, \ldots, t$. If each index $\left|H_{i+1}: H_{i}\right|(i=0,1, \ldots, t-1)$ is a prime power, then $G$ is an $E_{\pi}$-group, for any set $\pi$ of primes.

Corollary 1.2. Suppose that $G$ has a subgroup series

$$
1=H_{0} \leq H_{1} \leq \ldots \leq H_{t-1} \leq H_{t}=G
$$

and a supplement

$$
1=T_{t} \leq T_{t-1} \leq \ldots \leq T_{1} \leq T_{0}=G
$$

of this series in $G$ such that $H_{i}$ permutes with all Sylow subgroups of $T_{i}$ for all $i=1,2, \ldots, t$. If each index $\left|H_{i+1}: H_{i}\right|$ is a prime power, then $G$ is soluble.

Theorem C. Suppose that $G$ has a subgroup series

$$
1=H_{0}<H_{1} \leq \ldots \leq H_{t-1} \leq H_{t}=G
$$

and a subgroup $T$ such that $G=H_{1} T$ and $H_{i}$ permutes with all subgroups of $T$ for all $i=1,2, \ldots, t$. If each index $\left|H_{i+1}: H_{i}\right|$ is either a $\pi$-number or a $\pi^{\prime}$-number, then $G$ is a $C_{\pi}$-group and a $C_{\pi^{\prime}}$-group.

The following example shows that, under the conditions of Theorems A, B or C, the group $G$ is not necessarily $\pi$-separable.

Example 1.1. Let $G=A_{5} \times C_{7}$, where $C_{7}$ is a group of order 7 and $A_{5}$ is the alternating group of degree 5 . Let $C_{5}$ be a Sylow 5 -subgroup of $A_{5}$. Consider the subgroup series

$$
1=H_{0}<H_{1}<H_{2}<H_{3}=G,
$$

where $H_{1}=A_{4}$ and $H_{2}=A_{5}$. Then the series $1=T_{3}<T_{2}<T_{1}<T_{0}=G$, where $T_{2}=C_{7}$ and $T_{1}=C_{5} \times C_{7}$, is a supplement of Series $(* * *)$ in $G$. It is clear also that $H_{i}$ permutes with all subgroups of $T_{i}$, for all $i$. Let $\pi=\{5,7\}$. Then every index of Series $(* * *)$ is either a $\pi$-number or a $\pi^{\prime}$-number. However, $G$ is not $\pi$-separable.

\section{Preliminaries}

In this section, we cite some known results which are used in our proofs.

Lemma 2.1 (S. A. Cunihin [2, Theorem 1.4.2]). Let $N$ be a normal subgroup of $G$. If $N$ and $G / N$ are $C_{\pi}$-groups, then $G$ is a $C_{\pi}$-group.

Lemma 2.2 (O. Kegel [8, Theorem 3]). Let $A$ and $B$ be subgroups of $G$ such that $G \neq A B$ and $A B^{x}=B^{x} A$, for all $x \in G$. Then $G$ has a proper normal subgroup $N$ such that either $A \leq N$ or $B \leq N$.

Let $A$ be a subgroup of $G$. A subgroup $T$ is said to be a minimal supplement of $A$ in $G$ if $A T=G$ but $A T_{0} \neq G$ for all proper subgroups $T_{0}$ of $G$.

The following lemma is obvious.

Lemma 2.3. If $N$ is normal in $G$ and $T$ is a minimal supplement of $N$ in $G$, then $N \cap T \leq \Phi(T)$. 
Lemma 2.4 (P. Hall [7]). Suppose that $G$ has a Hall $p^{\prime}$-subgroup for each prime $p$ dividing $|G|$. Then $G$ is soluble.

Let $A$ and $B$ be subgroups of $G$ and $\emptyset \neq X \subseteq G$. Following [4, we say that $A$ is $X$-permutable (or $A X$-permutes) with $B$ if $A B^{x}=B^{x} A$ for some $x \in X$.

The following lemma is also evident.

Lemma 2.5. Let $A, B, X$ be subgroups of $G$ and $K \unlhd G$. If $A$ is $X$-permutable with $B$, then $A K / K$ is $X K / K$-permutable with $B K / K$ in $G / K$.

Lemma 2.6 (O. Kegel [9, Theorem 3]). If a subgroup $A$ of $G$ permutes with all Sylow subgroups of $G$, then $A$ is subnormal in $G$.

Lemma 2.7 (H. Wielandt [1]). If a $\pi$-subgroup $A$ of $G$ is subnormal in $G$, then $A \leq O_{\pi}(G)$.

\section{Proofs of Theorems A And A*}

Theorem A is a special case (when $X=1$ ) of the following theorem.

Theorem 3.1. Let $X$ be a normal $\pi$-separable subgroup of $G$ and $A$ a Hall $\pi$ subgroup of $G$. Let $G=A T$ for some subgroup $T$ of $G$, and let $q$ be a prime. If $A$ is $X$-permutable with every Sylow $p$-subgroup of $T$ for all primes $p \neq q$ and either $A$ is soluble or every $\pi^{\prime}$-subgroup of $T$ is soluble, then $T$ contains a complement of $A$ in $G$ and any two complements of $A$ in $G$ are conjugate.

Proof. Suppose that this theorem is false and let $G$ be a counterexample of minimal order. Then, clearly, $T$ is not a subgroup of $G$ with prime power order and $\left|\pi^{\prime}\right| \geq 2$. We now proceed with the proof via the following steps.

(1) $X=1$.

Suppose that $X \neq 1$ and let $D$ be a minimal normal subgroup of $G$ contained in $X$. Then $D$ is either a $\pi$-group or a $\pi^{\prime}$-group. In the former case we have $D \leq A$. Otherwise, $D \leq T$. We first claim that the hypothesis is still true for $G / D$. Clearly, $G / D=(A D / D)(T D / D)$, where $A D / D$ is a Hall $\pi$-subgroup of $G / D$ and $X / D$ is a normal $\pi$-separable subgroup of $G / D$. Moreover, if $A$ is soluble, then $A D / D \simeq A /(A \cap D)$ is soluble. Suppose that every $\pi^{\prime}$-subgroup of $T$ is soluble. Let $V / D$ be a $\pi^{\prime}$-subgroup of $T D / D$. Then $V=V \cap T D=D(V \cap T)$. Since $V / D=D(V \cap T) / D \simeq(V \cap T) /(V \cap T \cap D),(V \cap T) /(V \cap T \cap D)$ is a $\pi^{\prime}$-group. If $D$ is a $\pi^{\prime}$-group, then $D \leq T$ and so $V$ is a $\pi^{\prime}$-subgroup of $T$. Hence $V$ is soluble and thereby $V / D$ is soluble. Now assume that $D$ is a $\pi$-group. Then $V \cap T=[V \cap T \cap D] E$ for a Hall $\pi^{\prime}$-subgroup $E$ of $V \cap T$ by the Schur-Zassenhaus Theorem. Since $E$ is soluble by hypothesis, $V / D$ is soluble. Thus every $\pi^{\prime}$-subgroup of $T D / D$ is soluble. Now let $Q / D$ be a Sylow $p$-subgroup of $T D / D$, where $p \neq q$. Then for some Sylow $p$-subgroup $P$ of $T$, we have $Q / D=D P / D$. By hypothesis, $A X$-permutes with $P$. Hence, $A D / D$ is $X D / D$-permutable with $Q / D=D P / D$ in $G / D$ by Lemma 2.5. Therefore, our claim holds.

Since $|G / D|<|G|$, the minimal choice implies that $T D / D$ contains a complement $V / D$ of $A D / D$ in $G / D$ and every two complements of $A D / D$ in $G / D$ are conjugate. Obviously, $V / D=(V \cap T D) / D=D(V \cap T) / D \simeq(V \cap T) /(V \cap T \cap D)$. Since $A D / D$ is a Hall $\pi$-subgroup of $G / D, V / D$ is a Hall $\pi^{\prime}$-subgroup of $G / D$. If $D$ is a $\pi^{\prime}$-group, then $V$ is a Hall $\pi^{\prime}$-subgroup of $G$. Hence $V$ is a complement of $A$ in $G$. If $D$ is a $\pi$-group, then by the Schur-Zassenhaus Theorem, $V \cap T=[V \cap T \cap D] E$, 
for a Hall $\pi^{\prime}$-subgroup $E$ of $V \cap T$. It follows that $V=D(V \cap T)=D E$ and so $G=A E$. Thus, $E$ is a complement of $A$ in $G$ since $E$ is a Hall $\pi^{\prime}$-subgroup of $G$.

Now let $T_{1}$ and $T_{2}$ be Hall $\pi^{\prime}$-subgroups of $G$, where $T_{2} \leq T$. Then $T_{1} D / D=$ $T_{2}{ }^{x} D / D$, for some $x \in G$. If $D$ is a $\pi^{\prime}$-group, then $T_{1}=T_{2}{ }^{x}$, which contradicts the choice of $G$. Hence $D$ is a $\pi$-group. By hypothesis, either $D$ or $T_{2}{ }^{x}$ is soluble. Therefore, by the Schur-Zassenhaus Theorem, $T_{1}$ and $T_{2}{ }^{x}$ are conjugate in $T_{1} D$. This implies that every Hall $\pi^{\prime}$-subgroup of $G$ is conjugate with $T_{2}$ and hence every two complements of $A$ in $G$ are conjugate, which contradicts the choice of $G$.

(2) $O_{\pi}(G)=1$ and $O_{\pi^{\prime}}(G)=1$ (see the proof of (1)).

(3) A permutes with every Sylow $p$-subgroup $P$ of $G$, for all $p \neq q$ such that a Sylow p-subgroup of $T$ is a Sylow p-subgroup of $G$.

Since a Sylow $p$-subgroup $T_{p}$ of $T$ is a Sylow $p$-subgroup of $G, P=T_{p}{ }^{x}$, for some $x \in G$. Because $G=A T, x=t a$, where $a \in A$ and $t \in T$. Since $A$ permutes with the Sylow subgroup $T_{p}{ }^{t}$ of $T$, we obtain that $A$ permutes with $T_{p}{ }^{x}=P$.

(4) $G$ is not simple.

Let $P$ be any Sylow $p$-subgroup of $G$, where $q \neq p \in \pi^{\prime}$. Then by (2), $A P^{x}=$ $P^{x} A$, for all $x \in G$. Besides, $A P \neq G$ since $\left|\pi^{\prime}\right| \geq 2$. Hence $G$ is not simple by Lemma 2.2 .

(5) $T$ has a Hall $\pi^{\prime}$-subgroup.

Suppose that it is false. Then $D=A \cap T \neq 1$. Obviously, $T=D T$. Since $A$ is a Hall $\pi$-subgroup of $G, D$ is a Hall $\pi$-subgroup of $T$. Let $P$ be a Sylow $p$-subgroup of $T$, where $p \neq q$. Since $A P=P A$ by $(1),(A \cap T) P=A P \cap T=P A \cap T=P(A \cap T)$. Hence the hypothesis holds for $(D, T)$. If $T \neq G$, then $T$ is a $C_{\pi^{\prime}}$-group by the choice of $G$. In particular, $T$ has a Hall $\pi^{\prime}$-subgroup $E$, which, evidently, is a Hall $\pi^{\prime}$-subgroup $G$.

Now assume that $T=G$. First suppose that $A$ is a $q$-group. Let $D$ be a proper normal subgroup of $G$. We show that $D$ is a $C_{\pi}$-group. Let $p \neq q$ be a prime dividing $|D|, P$ a Sylow $p$-subgroup of $D$ and $G_{p}$ a Sylow $p$-subgroup of $G$ containing $P$. Then by hypothesis, $A G_{p}=G_{p} A$. Hence, $A G_{p} \cap D$ is a Hall $\{q, p\}$-subgroup of $D$. Besides, $A \cap D$ is a Sylow $q$-group of $D$ and $P=G_{p} \cap D$. Since $(A \cap D) P \leq A G_{p} \cap D$ and $\left|A G_{p} \cap D\right|=|A \cap D||P|$, we have that $(A \cap D) P=A G_{p} \cap D=P(A \cap D)$. Therefore the hypothesis holds for $D=(A \cap D) D$. This implies that $D$ is a $C_{\pi^{\prime}-}$ group by the choice of $G$. Let $D_{\pi^{\prime}}$ be a Hall $\pi^{\prime}$-subgroup of $D$. By the Frattini argument, $G=D N$, where $N=N_{G}\left(D_{\pi^{\prime}}\right)$. It follows from $|G: N|=|D: N \cap D|$ that $|G: N|=q^{a}$. Let $A_{0}$ be a Sylow $q$-subgroup of $N$. Since $T=G$, by (1), $A$ permutes with any Sylow $p$-subgroup $P$ of $G$, where $p \neq q$. Hence $A^{x}$ also permutes with all Sylow $p$-subgroups of $G$, where $p \neq q$. We may, therefore, assume that $A_{0} \leq A$. Then $A \cap N=A_{0}$ and, clearly, the hypothesis holds on $N=A_{0} N$. In view of (2), $N \neq G$. Hence $N$ is a $C_{\pi^{\prime}}$-group by the choice of $G$. Let $E$ be a Hall $\pi^{\prime}$-subgroup of $N$. Then, evidently, $E$ is also a Hall $\pi^{\prime}$-subgroup of $G$ since $|G: N|=q^{a}$.

Now let $T_{1}$ and $T_{2}$ be Hall $\pi^{\prime}$-subgroups of $G$. Then $D_{1}=T_{1} \cap D$ and $D_{2}=T_{2} \cap D$ are Hall $\pi^{\prime}$-subgroups of $D$. Hence $D_{1}$ and $D_{2}$ are conjugate in $D$. It follows that $N_{G}\left(D_{1}\right)=N_{G}\left(D_{2}\right)^{x}$ for some $x \in G$. Since $T_{1} \leq N_{G}\left(D_{1}\right)$ and $T_{2} \leq N_{G}\left(D_{2}\right), T_{1}$ is a conjugate of some Hall $\pi^{\prime}$-subgroup of $N_{G}\left(D_{2}\right)$. Hence $T_{1}$ and $T_{2}$ are conjugate in $G$. This contradiction shows that $A$ is not a Sylow $q$-subgroup of $G$. Let $P$ be any Sylow $p$-subgroup of $G$, where $p \neq q$ is a prime dividing $|A|$. Since $T=G$, by (2), $A P^{x}=P^{x} A=A$, for all $x \in G$. Hence $P^{G} \leq A$, which contradicts (2). 


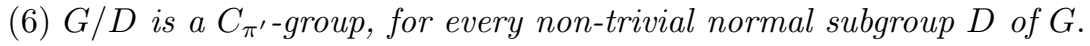

In view of (5), we may, without loss of generality, assume that $T$ is a Hall $\pi^{\prime}$ subgroup of $G$. Hence, as in the proof of (1), we obtain that $G / D$ satisfies the hypothesis. The minimal choice of $G$ implies that $G / D$ is a $C_{\pi^{\prime}}$-group.

(7) Every proper normal subgroup $D$ of $G$ is a $C_{\pi^{\prime}}$-group.

By (5), we may assume that $G=A T$, where $A$ is a Hall $\pi$-subgroup and $T$ is a Hall $\pi^{\prime}$-subgroup. Then $D=(D \cap A)(D \cap T)$. Let $p \neq q$ be a prime dividing $|T \cap D|$, $P$ a Sylow $p$-subgroup of $T \cap D$ and $G_{p}$ a Sylow $p$-subgroup of $G$ containing $P$. Then by hypothesis, $A G_{p}=G_{p} A$. Hence $A G_{p} \cap D$ is a Hall $\pi \cup\{p\}$-subgroup of $D$. Besides, $A \cap D$ is a Hall $\pi$-subgroup of $D$ and $P=G_{p} \cap D$. Since $(A \cap D) P \leq A G_{p} \cap D$ and $\left|A G_{p} \cap D\right|=|A \cap D||P|,(A \cap D) P=A G_{p} \cap D=P(A \cap D)$. Therefore the hypothesis holds for $D=(A \cap D)(D \cap T)$. The minimal choice implies that $D$ is a $C_{\pi^{\prime}}$ group.

Final contradiction. By (4), $G$ has a proper normal subgroup $D \neq 1$. By (6) and (7) both $D$ and $G / D$ are $C_{\pi^{\prime}}$-groups. Hence $G$ is a $C_{\pi^{\prime}}$-group by Lemma 2.1 . The final contradiction completes the proof.

Proof of Theorem $A^{*}$. In view of the Feit-Thompson Theorem about solvability of groups of odd order, we know that either every $\pi$-group or every $\pi^{\prime}$-group is soluble. Hence Theorem A* is a corollary of Theorem A.

\section{Proof of Theorem B}

Theorem B is a special case (when $X=1$ ) of the following theorem.

Theorem 4.1. Let $X$ be a normal $\pi$-separable subgroup of $G$. Suppose that $G$ has a subgroup series

$$
1=H_{0} \leq H_{1} \leq \ldots \leq H_{t-1} \leq H_{t}=G
$$

and a supplement

$$
1=T_{t} \leq T_{t-1} \leq \ldots \leq T_{1} \leq T_{0}=G
$$

of this series in $G$ such that $H_{i} X$-permutes with every Sylow subgroup of $T_{i}$ for all $i=1,2, \ldots, t$. If each index $\left|H_{i+1}: H_{i}\right|$ is either a $\pi$-number or a $\pi^{\prime}$-number, then $G$ is an $E_{\pi}$-group and an $E_{\pi^{\prime}}$-group. Moreover, if each $\pi$-index $\left|H_{i+1}: H_{i}\right|$ is a prime power, then $G$ has a soluble Hall $\pi$-subgroup.

Proof. Suppose that this theorem is false and let $G$ be a counterexample of minimal order. Without loss of generality, we may assume that $H_{1} \neq 1$. We proceed with the proof by proving the following claims:

(1) The assertions of the theorem hold for every non-trivial quotient $G / N$ of $G$. We consider the series

$$
1=H_{0} N / N \leq H_{1} N / N \leq \ldots \leq H_{t-1} N / N \leq H_{t} N / N=G / N
$$

and its supplement

$$
1=T_{t} N / N \leq T_{t-1} N / N \leq \ldots \leq T_{1} N / N \leq T_{0} N / N=G / N
$$

in $G / N$. By Lemma 2.5, $H_{i} N / N$ is $X N / N$-permutable with any Sylow subgroup of $T_{i} N / N$ for all $i=1,2, \ldots, t$. On the other hand, since $\left|H_{i+1} N / N: H_{i} N / N\right|=$ $\left|H_{i+1} N: H_{i} N\right|=\left|H_{i+1}: H_{i}\right|:\left|N \cap H_{i+1}: N \cap H_{i}\right|$, every index of the series (1) is either a $\pi$-number or a $\pi^{\prime}$-number (a $\pi$-prime power or a $\pi^{\prime}$-number). Moreover, obviously, $X N / N \simeq X /(X \cap N)$ is $\pi$-separable. This shows that the hypothesis 
holds on $G / N$. Hence in the case $N \neq 1$, the assertions of the theorem hold for $G / N$ by the choice of $G$.

(2) $O_{\pi^{\prime}}(G)=1=O_{\pi}(G)$.

Suppose that $D=O_{\pi^{\prime}}(G) \neq 1$. Then by (1), $G / D$ has a Hall $\pi^{\prime}$-subgroup $A / D$ and a Hall $\pi$-subgroup $B / D$. Then, obviously, $A$ is a Hall $\pi^{\prime}$-subgroup of $G$. By the Schur-Zassenhaus Theorem, $D$ has a complement $V$ in $B$, which, clearly, is a Hall $\pi$-subgroup of $G$. Hence $G$ is an $E_{\pi^{-}}$-group and an $E_{\pi^{\prime}}$-group. Besides, if every $\pi$-index of series (1) is a prime power, then $B / D$ has a soluble Hall $\pi$-subgroup $B / D$. It follows that $V$ is also soluble. This contradiction shows that $O_{\pi^{\prime}}(G)=1$. Analogously, we may prove that $O_{\pi}(G)=1$.

(3) $X=1$.

Indeed, if $N$ is a minimal normal subgroup of $G$ contained in $X$, then $N$ is either a $\pi$-group or a $\pi^{\prime}$-group, which contradicts (2).

(4) $T_{1} \neq G$.

Suppose that $T_{1}=G$. Then by hypothesis and (3), $H_{1}$ permutes with all Sylow subgroups of $G$. It follows from Lemma 2.6 that $H_{1}$ is subnormal in $G$. Since $H_{1}$ is either a $\pi$-group or a $\pi^{\prime}$-group, $H_{1} \leq O_{\pi}(G)$ or $H_{1} \leq O_{\pi^{\prime}}(G)$ by Lemma 2.7. It follows from (2) that $H_{1}=1$, which contradicts $H_{1} \neq 1$. Hence (4) holds.

(5) The assertions of the theorem hold for $T_{1}$.

We consider the series

$$
1=H_{0} \cap T_{1} \leq H_{1} \cap T_{1} \leq \ldots \leq H_{t-1} \leq H_{t} \cap T_{1}=T_{1} .
$$

Then the series

$$
1=T_{t} \leq T_{t-1} \leq \ldots \leq T_{1}
$$

is a supplement of the series (2) in $T_{1}$ since $\left(H_{i} \cap T_{1}\right) T_{i}=H_{i} T_{i} \cap T_{1}=G \cap T_{1}=T_{1}$. Since $H_{i+1}=H_{i} T_{1} \cap H_{i+1}=H_{i}\left(H_{i+1} \cap T_{1}\right),\left|H_{i+1}: H_{i}\right|=\left|H_{i+1} \cap T_{1}: H_{i} \cap T_{1}\right|$, for all $i=1,2, \ldots, t-1$ and $\left|H_{1} \cap T_{1}: H_{0} \cap T_{1}\right|=\left|H_{1} \cap T_{1}\right| \leq\left|H_{1}: 1\right|$, we see that every index of the series (2) is either a $\pi$-number or a $\pi^{\prime}$-number. Moreover, if every $\pi$-index of the series (1) is a prime power, then every $\pi$-index of the series (2) is a prime power. Now let $E$ be a Sylow subgroup of $T_{i}$. By (3) and the hypothesis, $H_{i} E=E H_{i}$. Hence $H_{i} E \cap T_{1}=E\left(H_{i} \cap T_{1}\right)=\left(H_{i} \cap T_{1}\right) E$. This shows that the hypothesis holds for $T_{1}$. The minimal choice of $G$ implies that (5) holds.

Final contradiction. Let $\left(T_{1}\right)_{\pi}$ and $\left(T_{1}\right)_{\pi^{\prime}}$ be a Hall $\pi$-subgroup and a Hall $\pi^{\prime}$ subgroup of $T_{1}$, respectively. By (3) and the hypothesis, $H_{1}$ permutes with all Sylow subgroups of $\left(T_{1}\right)_{\pi}$. Hence $H_{1}$ permutes with $\left(T_{1}\right)_{\pi}$. Similarly, $H_{1}$ permutes with $\left(T_{1}\right)_{\pi^{\prime}}$. By hypothesis, $H_{1}$ is either a $\pi$-group or a $\pi^{\prime}$-group. Assume that $H_{1}$ is a $\pi$-group. Since $G=H_{1} T_{1}$, we see that $G_{\pi}=H_{1}\left(T_{1}\right)_{\pi}$ is a Hall $\pi$-subgroup of $G$ and $\left(T_{1}\right)_{\pi^{\prime}}$ is a Hall $\pi^{\prime}$-subgroup of $G$. If $H_{1}$ is a $\pi^{\prime}$-group, then $G_{\pi^{\prime}}=H_{1} T_{\pi^{\prime}}$ is a Hall $\pi^{\prime}$-subgroup of $G$ and $T_{\pi}$ is a Hall $\pi$-subgroup of $G$. Finally, we prove that if every $\pi$-index of the series (1) is a prime power, then $G$ has a soluble Hall $\pi$-subgroup. In fact, by (5), we see that $\left(T_{1}\right)_{\pi}$ is soluble. If $H_{1}$ is a $\pi^{\prime}$-group, then $\left(T_{1}\right)_{\pi}$ is a soluble Hall $\pi$-subgroup of $G$ since $G=H_{1} T_{1}$. If $H_{1}$ is a $p$-group, then $H_{1}\left(T_{1}\right)_{\pi}$ is a Hall $\pi$-subgroup of $G$. Since $\left(T_{1}\right)_{\pi}$ is soluble and $H_{1}$ permutes with every Sylow subgroup of $\left(T_{1}\right)_{\pi}$, we see that $H_{1}\left(T_{1}\right)_{\pi}$ is soluble by Lemma 2.4. The contradiction completes the proof. 


\section{Proof of Theorem $\mathrm{C}$}

Theorem $\mathrm{C}$ is a special case (when $X=1$ ) of the following theorem.

Theorem 5.1. Let $X$ be a normal $\pi$-separable subgroup of $G$. Suppose that $G$ has a subgroup series

$$
1=H_{0}<H_{1} \leq \ldots \leq H_{t-1} \leq H_{t}=G
$$

where $H_{1} \neq 1$, and a subgroup $T$ such that $T H_{1}=G$ and $H_{i} X$-permutes with all nilpotent subgroups of $T$ for all $i=1,2, \ldots, t$. If the index $\left|H_{i+1}: H_{i}\right|$ is either a $\pi$-number or a $\pi^{\prime}$-number, then $G$ is a $C_{\pi}$-group and a $C_{\pi^{\prime}}$-group as well.

Proof. Suppose that this theorem is false and let $G$ be a counterexample of minimal order. By Theorem 3.2, $G$ has a Hall $\pi$-subgroup $S$ and a Hall $\pi^{\prime}$-subgroup $J$. Hence we may assume that either some Hall $\pi$-subgroup $S_{1}$ of $G$ is not conjugate with $S$ or some Hall $\pi^{\prime}$-subgroup $J_{1}$ of $G$ is not conjugate with $J$. We may, without loss of generality, assume that $2 \notin \pi$. Then $S$ is soluble by the Feit-Thompson Theorem of groups of odd order. We proceed with the proof via the following steps.

(1) The assertion of the theorem holds for every non-trivial quotient $G / N$ of $G$. Consider the series

$$
1=H_{0} N / N \leq H_{1} N / N \leq \ldots \leq H_{t-1} N / N \leq H_{t} N / N=G / N
$$

of $G / N$. Then $\left(H_{1} N / N\right)(T N / N)=G / N$. Let $V / N$ be any nilpotent subgroup of $T N / N$. Then $V=N(V \cap T)$ and so $V / N \simeq V \cap T / V \cap T \cap N$. Let $V_{0}$ be a minimal supplement of $V \cap T \cap N$ in $V \cap T$. Then by Lemma 2.3, $V_{0}$ is nilpotent. Hence $H_{i} X$ permutes with $V_{0}$ by hypothesis. Besides, $V / N=N(V \cap T)=N V_{0}(V \cap T \cap N) / N=$ $N V_{0} / N$. Hence by Lemma $2.5, H_{i} N / N$ is $X N / N$-permutable with any nilpotent subgroup of $T N / N$, for all $i=1,2, \ldots, t$. This shows that the hypothesis holds for $G / N$. Hence, in the case $N \neq 1$, the assertion of the theorem holds for $G / N$ by the choice of $G$.

(2) $O_{\pi^{\prime}}(G)=1=O_{\pi}(G)$.

Suppose that $D=O_{\pi^{\prime}}(G) \neq 1$. Then by (1), there are elements $x, y \in G$ such that $S_{1}{ }^{x} D=S D$ and $J_{1}{ }^{y} D=J D$. Since $S D / D \simeq S$ is soluble, by the SchurZassenhaus Theorem, $S_{1}{ }^{x}$ and $S$ are conjugate in $S D$. On the other hand, since $D \subseteq J, J_{1}^{y}=J$. This contradiction shows that $O_{\pi^{\prime}}(G)=1$. Analogously, we can prove that $O_{\pi}(G)=1$.

(3) $X=1$.

Indeed, if $N$ is a minimal normal subgroup of $G$ contained in $X$, then $P$ is either a $\pi$-group or a $\pi^{\prime}$-group, which contradicts (2).

(4) $T \neq G$ (see the proof of (4) in the proof of Theorem 3.2).

(5) The assertions of the theorem hold for $T$ (see the proof of (5) in the proof of Theorem 3.2).

(6) If $D$ is a normal subgroup of $G$ and $H_{1} \leq D$, then $D=G$.

Suppose that $D \neq G$. Let $D_{i}=H_{i} \cap D$, for all $i=1,2, \ldots, t$. Consider the series

$$
1=D_{0} \leq D_{1} \leq \ldots \leq D_{t-1} \leq D_{t}=D
$$

First note that $D=D \cap H_{1} T=H_{1}(D \cap T)$. Let $E$ be a nilpotent subgroup of $D \cap T$. Then $H_{i} E=E H_{i}$ and so $H_{i} E \cap D=E\left(H_{i} \cap D\right)=E D_{i}=D_{i} E$. Thus $D_{i}$ permutes with every nilpotent subgroup of $D \cap T$ for all $i=1,2, \ldots, t-1$. On the other hand, $\left|D_{i}: D_{i-1}\right|=\left|\left(D \cap H_{i}\right) H_{i-1}: H_{i-1}\right||| H_{i}: H_{i-1} \mid$. Hence each index $\left|D_{i}: D_{i-1}\right|$ is either a $\pi$-number or a $\pi^{\prime}$-number. Therefore $D$ is a $C_{\pi^{-} \text {-group }}$ 
and a $C_{\pi^{\prime}}$-group by the choice of $G$. Since $1 \neq H_{1} \leq D, G / D$ is a $C_{\pi^{-}}$-group and a $C_{\pi^{\prime}}$-group by (1) and the choice of $G$. It follows from Lemma 2.1 that $G$ is a $C_{\pi^{-}}$-group and a $C_{\pi^{\prime}}$-group, which contradicts the choice of $G$. Hence, (6) holds.

(7) If $H_{1}$ is a $\pi$-group ( $\pi^{\prime}$-group) and $D$ is a normal subgroup of $G$ containing a Hall $\pi$-subgroup of $T$ (containing a Hall $\pi^{\prime}$-subgroup of $T$, respectively), then the hypothesis holds for D.

Suppose, for example, that $H_{1}$ is a $\pi$-group. We claim that $D=\left(H_{1} \cap D\right)(T \cap D)$. In fact, let $E$ be a Hall $\pi$-subgroup of $T$ contained in $D$ and $T_{\pi^{\prime}}$ a Hall $\pi^{\prime}$-subgroup of $T$. Since $H_{1}$ is a $\pi$-group and $G=H_{1} T, T_{\pi^{\prime}}$ is also a Hall $\pi^{\prime}$-subgroup of $G$. Clearly $H_{1} E$ is a Hall $\pi$-subgroup of $G$. Hence $D=\left(D \cap H_{1} E\right)\left(D \cap T_{\pi^{\prime}}\right)=$ $E\left(D \cap H_{1}\right)\left(D \cap T_{\pi^{\prime}}\right)=\left(D \cap H_{1}\right)\left(E\left(D \cap T_{\pi^{\prime}}\right)\right)=\left(D \cap H_{1}\right)(T \cap D)$. Thus, our claim holds. Now, by similar inference as in (6), we see that the hypothesis holds for $D$.

(8) If $H_{1}$ is a $\pi$-group ( $\pi^{\prime}$-group) and $E$ is a Hall $\pi$-subgroup of $T$ (a Hall $\pi^{\prime}$ subgroup of $T)$, then $E^{G} \neq G$.

Assume, for example, that $H_{1}$ is a $\pi$-group. Since $G=H_{1} T$, we have that $x=h t$, where $h \in H_{1}$ and $t \in T$, for any $x \in G$. Because $H_{1}$ permutes with all Sylow subgroups of $T, H_{1} E^{t}=E^{t} H_{1}$. Hence $H_{1} E^{x}=H_{1} E^{t h}=E^{t h} H_{1}$. Now by Lemma 2.2, either $H_{1}{ }^{G} \neq G$ or $E^{G} \neq G$. But in view of (7), the former case is impossible. Hence $E^{G} \neq G$.

Final contradiction. In view of $(1),(7),(8)$ and Lemma $2.1, G$ is a $C_{\pi}$-group and a $C_{\pi^{\prime}}$-group. The contradiction completes the proof.

Remark. We prove Theorem C on the base of the Feit-Thompson Theorem of groups of odd order. The following fact may be proved without using this deep result.

Theorem. Suppose that $G$ has a subgroup series

$$
1=H_{0}<H_{1} \leq \ldots \leq H_{t-1} \leq H_{t}=G
$$

and a subgroup $T$ such that $G=H_{1} T$ and $H_{i}$ permutes with all subgroups of $T$ for all $i=1,2, \ldots, t$. If each index $\left|H_{i+1}: H_{i}\right|$ is either a $\pi$-number or a $\pi^{\prime}$-number, then $G$ is an $E_{\pi}$-group and an $E_{\pi^{\prime}}$-group. Moreover, if each $\pi$-index $\left|H_{i+1}: H_{i}\right|$ is a prime power, then $G$ is a $C_{\pi}$-group and a $C_{\pi^{\prime}}$-group.

\section{ACKNOWLEDGMENT}

The authors are very grateful for the helpful suggestions of the referee.

\section{REFERENCES}

[1] S. A. Čunihin, On theorems of Sylow's type, Doklady Akad. Nauk. SSSR, 1949, 66, 165-168. MR0029903 (10:678a)

[2] S. A. Cunihin, Subgroups of finite groups, Nauka i Tehnika, Minsk, 1964. MR0212082 $(35: 2957)$

[3] Wenbin Guo, The Theory of Classes of Groups, Science Press, Kluwer Academic Publishers, Beijing-New York-Dordrecht-Boston-London, 2000. MR.1862683 (2003a:20029)

[4] Wenbin Guo, K. P. Shum and Alexander N. Skiba, $X$-semipermutable subgroups of finite groups, J. Algebra, 2007, 315, 31-41. MR2344332 (2008g:20039)

[5] Wenbin Guo, K. P. Shum and Alexander N. Skiba, Schur-Zassenhaus Theorem for Xpermutable subgroups, Algebra Colloquium, 2008, 15(2), 185-192. MR2400176|(2009b:20025)

[6] P. Hall, A note on soluble groups, J. London Math. Soc., 1928, 3, 98-105.

[7] P. Hall, A characteristic property of soluble groups, J. London Math. Soc., 1937, 12, 188-200.

[8] O. H. Kegel, Produkte nilpotenter Gruppen, Arch. Math., 1961, 12, 90-93. MR0133365 (24:A3199) 
[9] O. Kegel, Sylow-Gruppen and Subnormalteiler endlicher Gruppen, Math. Z., 1962, 78, 205221. MR0147527 (26:5042)

[10] D. J. S. Robinson, A Course in the Theory of Groups, Springer-Verlag, New York-HeidelbergBerlin, 1982. MR648604 (84k:20001)

[11] H. Wielandt, Subnormal subgroups and permutation groups. Lectures given at the Ohio State University, Columbus, Ohio, 1971.

Department of Mathematics, University of Science and Technology of China, Hefei 230026, People's Republic of China

E-mail address: wbguo@ustc.edu.cn

Department of Mathematics, Francisk Skorina Gomel State University, Gomel 246019, Belarus

E-mail address: alexander.skiba49@gmail.com 